\title{
Emociones: Desde la sociología a la neurociencia
}

\author{
[Emotions: From sociology to neuroscience] \\ Karina Rdz-Navarro ${ }^{1}$ (1), \& Mauricio González 2 (1) \\ ${ }^{1}$ Universidad de Chile; 2Universidad de La Serena
}

\section{In memoriam de Humberto Maturana (1928-2021).}

Todos, en algún momento en la vida, nos hemos dejado llevar por alguna emoción y hemos hecho algo motivados por ese impulso emocional. En una cultura que considera que lo racional como algo trascendente e inherentemente válido, las emociones parecen ser algo que surge de forma misteriosa y que debemos aprender a gestionar o a evitar. Una premisa similar orientó el trabajo científico en torno a las emociones durante muchos años. Desde los inicios de la ciencia moderna, las emociones fueron relegadas a un segundo plano, al ser consideradas acciones irracionales o como una categoría residual de análisis científico que parecía poco productiva (Asún, Rdz-Navarro, \& Tintaya, 2020; Tudor, 2003). Sin embargo, hacia mediados del siglo XX, comenzó a emerger un interés gradual y progresivo por estudiar las emociones en diferentes disciplinas, como la sociología, la psicología, la educación, o la neurociencia.

Los primeros esfuerzos por estudiar los fenómenos emocionales e incorporarlos como causa o consecuencia de otros fenómenos de interés para la ciencia fueron esfuerzos parcelados y disciplinares. Es decir, esfuerzos que incorporaban a las emociones como un "extra" del análisis más que como un elemento central y que involucraban a profesionales de una sola disciplina en lugar de equipos multidisciplinarios. Hoy en día, ambas cosas están cambiando.

La evidencia empírica acumulada a partir de los múltiples esfuerzos de investigación (para una revisión, ver e.g., Ariza, 2021; Bericat 2016; Dolan, 2002) ha mostrado con enorme claridad que el principal motivador de la acción -ya sea individual o colectiva- no es la razón como se creía hace tiempo sino la emoción (e.g., Flam \& King, 2005; Jasper, 2011; Reed, 2020). Tanto es así que se ha llegado incluso a afirmar que el origen de lo social es la emoción (Maturana, 1990/2020) o que el pegamento de lo social está en los fenómenos emocionales (Collins, 2004).

La emoción produce una poderosa influencia en la cognición (Beer, 2017). Contribuye en la fijación de creencias y a la facilitación del recuerdo de aquellas experiencias cotidianas que evocaron sentimientos de alegría, tristeza, placer, o sufrimiento. Las emociones detonan complejos estados fisiológicos, psicológicos, y sociales que permiten a los organismos sentir si los eventos del entorno son agradables o desagradables, placenteros o displacenteros. Este continuo marcado por dos polos, también denominados positivo y negativo, se denomina la valencia o valor de las emociones. La valencia de las emociones, la intensidad 
con la que se experimentan y la gran variedad de emociones que un ser humano puede llegar a experimentar en cortos períodos de tiempo o de manera simultánea (Asún, Rdz-Navarro, Zúñiga, et al., 2020) refleja la complejidad y riqueza del fenómeno, en el cual, una misma emoción dependiendo del contexto puede generar reacciones muy distintas de una persona a otra. Las emociones son simultáneamente fenómenos biológicos que pueden surgir de estímulos internos o externos, experiencias subjetivas, fenómenos construidos psicológica, social y culturalmente, motivadores o inhibidores de la conducta, y una forma de comunicación entre individuos que facilita o inhibe las interacciones sociales (Barrett, 2009; Jasper, 2018).

El que las emociones sean tantas cosas a la vez, e involucren fenómenos que escapan a los límites disciplinares de la sociología, la psicología, la biología, o la neurociencia, hace evidente que su estudio es de interés transdisciplinar y requiere de una aproximación multidisciplinar. Sólo mediante aproximaciones de este tipo, es posible comprender de manera integral los fenómenos emocionales en sí mismos, las variables que influyen en su generación y, la influencia de las emociones en otros fenómenos o procesos ya sean sociales, culturales, psicológicos, biológicos, o neurofisiológicos. El trabajo y diálogo multi, inter, y transdisciplinar en torno a los fenómenos emocionales está comenzando a dar sus frutos. En años recientes se han generado teorías novedosas con abundante evidencia empírica que estimulan controversias y debates apasionados, además de potenciar el desarrollo de nuevas investigaciones científicas (Alastuey, 2000; Zamora, \& Mantilla,
2017). Sin embargo, a pesar de estos avances, es evidente que en el campo de las emociones todavía falta mucho por hacer.

Convencidos de la productividad de incorporar las emociones en las distintas esferas de investigación de las ciencias sociales y de que la naturaleza del fenómeno emocional brinda un espacio privilegiado en el cual se desdibujan las fronteras disciplinares, emprendimos el desafío de editar este dossier con el objetivo de contribuir con la difusión de investigaciones latinoamericanas que abordasen los fenómenos emocionales para dar cuenta de distintos problemas de investigación. El resultado de estos meses de trabajo dio como fruto este volumen en el cual se congrega un conjunto de manuscritos que son reflejo de la diversidad temática, teórica, y metodológica del campo, así como de lo productivo que puede llegar a ser incorporar un enfoque de emociones al abordar los distintos objetos de estudio de interés de las ciencias sociales.

El primer artículo que presentamos en este volumen editado fue desarrollado por Asún, Palma, Aceituno y Duarte. El artículo combina el conocimiento de las distintas disciplinas de las cuales proviene cada uno de sus autores para dar cuenta del impacto emocional que ha tenido la pandemia del Covid-19 en la población juvenil. Este trabajo, no solo invita a una reflexión importante sobre los efectos de la pandemia, sino que sitúa a las emociones como su foco principal para explicar por qué los jóvenes han sido uno de los grupos más afectados por las consecuencias sociales de la pandemia. Por su parte, el trabajo de Poblete indaga las emociones experimentadas por profesoras durante su trabajo en la sala de clases, la forma 
en que ellas las interpretan, y las atribuciones que hacen sobre los eventos que las provocan. Este trabajo, al igual que el anterior, pone el acento en el fenómeno emocional, aunque desde una perspectiva un tanto distinta, pues a partir de sus hallazgos abre la puerta a una interesante reflexión sobre el rol que juegan las emociones en los procesos de enseñanza y cómo ellas pueden potenciar el compromiso docente. En una línea similar, el trabajo de Colin centra su mirada en la nostalgia sobre el barrio a través de una investigación interesante que explora las vivencias y discursos de varias generaciones de personas. A partir de la lectura del trabajo, es posible notar los cambios que han experimentado en los últimos años tres barrios de la ciudad de Valparaíso y comprender cómo la nostalgia se transforma en un catalizador de la construcción de memorias e identidades colectivas.

Así como las emociones pueden afectar nuestra vida cotidiana, nuestro compromiso laboral, o contribuir a la construcción de memorias e identidades colectivas, las emociones también pueden ayudarnos a elaborar nuestras propias teorías sobre aquello que ocurre en la realidad y, a su vez, las personas elaboramos teorías personales sobre los fenómenos emocionales. El trabajo de Cuadra y Castro aborda justamente este tema, centrando su foco en un aspecto relativamente olvidado de las teorías subjetivas: su dimensión emocional. Para quienes aún no conocen de este campo, la lectura de este trabajo será de gran utilidad pues en el manuscrito se presenta una interesante síntesis de las principales investigaciones que se han hecho sobre las teorías subjetivas, lo cual permite comenzar a dimensionar las múltiples posibilidades de investigación que emergen al considerar a los fenómenos emocionales dentro de este marco de trabajo.

Para que las emociones puedan cumplir los múltiples roles que hemos mencionado (y aquellos que se nos han quedado en el tintero), debemos ser capaces de reconocerlas y aprender a gestionarlas de forma saludable. La educación emocional ha sido por largo tiempo un punto olvidado de las políticas públicas en Chile y probablemente en el resto del mundo. Para subsanar este problema que probablemente está a la base de los múltiples problemas de salud mental de la población, el trabajo de Farkas propone usar los cuentos infantiles como una herramienta para el desarrollo emocional en la primera infancia. Este trabajo, si bien se centra en propuestas que han surgido principalmente desde el mundo de la psicología, encierra un interés transversal debido a que los procesos de socialización primaria son un elemento central de diversas disciplinas desde la educación, pasando por la sociología, la psicología e incluso áreas ligadas a la neurociencia.

Los dos trabajos que cierran este volumen ofrecen perspectivas bastante distintas a las que hemos comentado hasta ahora. El trabajo de Aracena y González invita al lector a hacer un recorrido por los debates teóricos más interesantes respecto a la naturaleza de las emociones a partir de la evidencia empírica, planteando un desafío importante a la investigación sobre emociones: la necesidad del desarrollo de investigaciones experimentales. El campo experimental ha alcanzado interesantes desarrollos en la psicología y la neurociencia, pero ocupa un plano secundario en otras disciplinas que también abordan fenómenos emocionales y que podrían 
beneficiarse de esta perspectiva metodológica. A modo de ejemplo, Aracena y González presentan los resultados de un estudio breve sobre los conceptos de emoción y los atributos que las personas asocian a estos conceptos en emociones básicas y secundarias. Finalmente, el trabajo de Weistein y Aldunate es una interesante revisión de estudios de diferentes campos ligados al mundo de las neurociencias, que permiten identificar el conocimiento acumulado respecto a los factores que influyen en el procesamiento de las agresiones verbales considerando a las emociones -especialmente a las emociones negativas y el daño emocional- como consecuencias de la interacción verbal con otras personas.
A partir de este conjunto de trabajos que, a modo de humilde homenaje al Prof. Dr. Humberto Maturana quien falleció mientras estábamos trabajando en este dossier, hemos denominado "Emociones: Desde la sociología a la neurociencia”, esperamos haber contribuido a la difusión de investigaciones en el campo de las emociones, y demostrado su potencial en tanto causas y consecuencias de fenómenos de interés para las diversas disciplinas que componen las ciencias sociales y del comportamiento. Esperamos que la lectura de los trabajos de este dossier aliente a los investigadores y a las nuevas generaciones de científicos sociales a incorporar las emociones en sus investigaciones.

\section{REFERENCIAS}

Asún, R. A., Rdz-Navarro, K., \& Tintaya, M. (2020). ¿Por qué surgen los estallidos sociales? Emociones, redes interpersonales, rituales y participación en protestas. Ultima Década, 28(54), 5-40. https://doi.org/10.4067/S071822362020000200005

Asún, R. A., Rdz-Navarro, K., Zúñiga, C., \& Louis, W. (2020). Modelling the mediating effect of multiple emotions in a cycle of territorial protests. Social Movement Studies, 1-19. https://doi.org/10.1080/14742837.2020.1867093

Alastuey, E. B. (2000). La sociología de la emoción y la emoción en la sociología. Papers: Revista de Sociología, 62(1), 145-176. https://doi.org/ $10.5565 /$ rev/papers/v62n0.1070
Ariza, M. (2021). The sociology of emotions in Latin America. Annual Review of Sociology, 47, 1-19. https://doi.org/10.1146/annurevsoc-021021-054653

Barrett, L. F. (2009). Variety is the spice of life: A psychological constructionist approach to understanding variability in emotion. Cognition \& Emotion, 23(7), 1284-1306. https://doi.org/10.1080/02699930902985894

Beer, J. S. (2017). Current emotion research in social neuroscience: How does emotion influence social cognition? Emotion Review, $9(2), 172-180$. https://doi.org/10.1177/1754073916650492 
Bericat, E. (2016). The sociology of emotions: Four decades of progress. Current Sociology, 64(3), 491-513. https://doi.org/10.1177\%2F00 11392115588355

Collins, R. (2004). Interaction ritual chains. Princeton University Press.

Damasio, A. (2018). The strange order of things: Life, feeling, and the making of cultures. Pantheon.

Dolan, R. J. (2002). Emotion, cognition, and behavior. Science, 298(5596), 1191-1194. https://doi.org/10.1126/science.1076358

Flam, H., \& King, D. (2007). Emotions and social movements. Routledge.

Jasper, J. M. (2011). Emotions and social movements: Twenty years of theory and research. Annual Review of Sociology, 37(1), 285-303. https://doi.org/10.1146/annurev-soc-081309-150015

Jasper, J. M. (2018). The emotions of protest. University of Chicago Press.
Maturana, H. (2020). Emociones y lenguaje en educación y política. Editorial Planeta (Trabajo original publicado en 1990).

Reed, J. P. (2020). Sandinista narratives: Religion, sandinismo, and emotions in the making of the Nicaraguan insurrection and revolution. Lexington Books.

Tudor, A. (2003). A (macro) sociology of fear? The Sociological Review, 51(2), 238-256. https://doi.org/10.1111/1467-954X.00417.

Zamora, R. L., \& Mantilla, L. (2017). Desde la sociología de las emociones a la crítica de la biopolítica. Revista Latinoamericana de Estudios Sobre Cuerpos, Emociones y Sociedad, 25(9), 24-33.

http://www.relaces.com.ar/index.php/relaces /article/view/139/136 